\title{
UNA HERRAMIENTA PARA LA PROMOCIÓN DE LA SALUD EN LA TERCERA EDAD: TECNOLOGÍA DE LA COMUNICACIÓN E INFORMACIÓN
}

\author{
A TOOL FOR HEALTH PROMOTION IN \\ THE THIRD AGE: INFORMATION AND \\ COMMUNICATION TECHNOLOGY
}

\section{UMA FERRAMENTA PARA A PROMOÇÃO DA SAÚDE NA TERCEIRA IDADE: A TECNOLOGIA DE INFORMAÇÃO E COMUNICAÇÃO}

\author{
Sagrário Gómez Cantarino ${ }^{1}$ \\ María Idoia Ugarte Gurrutxaga ${ }^{2}$ \\ Maria Del Carmen Bouzas Mosquera ${ }^{3}$
}

Como citar este artículo: Cantarino SG, Gurrutxaga MIU, Mosquera MDCB. Una herramienta para la promoción de la salud en la tercera edad: tecnología de la comunicación e información. Rev baiana enferm. 2020;34:e35762.

El envejecimiento a nivel poblacional afecta a la totalidad de la sociedad, cuestión que se extrapola a nivel global. Actualmente en nuestra cultura occidental, esta situación es habitual, pero en la historia reciente esta cuestión era impensable, puesto que solo se alcanzaba la edad adulta en una proporción minoritaria de la población ${ }^{(1)}$.

Se estima que a nivel mundial, aproximadamente en el año 2050, se tripliquen las personas mayores de 65 años, reflejándose en unas cifras aproximadas de unos mil quinientos millones de habitantes ${ }^{(1)}$.

Por tanto, el interés del tema viene dada por la necesidad de analizar las actividades de ocio en la población anciana en relación al manejo de las nuevas tecnologías de la información (TIC) y la implicación de las mismas para la salud. Por tanto, con esta revisión se contribuirá al conocimiento en la etapa de la vejez de la influencia de las TIC en relación al aumento actividades sociales diarias, y por ende, de su salud.

Las personas que actualmente conforman el estrato social de la tercera edad, provienen de una época reciente en la que no se disponía de innovaciones tecnológicas tales como la telefonía móvil, internet e incluso los mensajes de texto en el propio aparato de telefonía, actualmente extendidos ampliamente a nivel social. En la época donde se desarrollaron y formaron nuestros mayores, no se contaba con agendas electrónicas, ordenadores o telefonía móvil. Su mundo estaba constituido por las relaciones

Enfermera. Doctorado en Campo de Sexualidad y Mujer. Universidad Cartilla-La Mancha. Toledo, España. sagrario.gomez@uclm.es. https://orcid.org/0000-00029640-0409.

Enfermera. Doctorado. Profesora Colaboradora. Universidad Cartilla-La Mancha. Toledo, España. https://orcid.org/0000-0003-24I3-3628.

3 Enfermera. Doctorado en Ciencias de la Enfermería. Profesora Ayudante. Universidad Cartilla-La Mancha. Toledo, España. https://orcid.org/0000-0003-I884- I820. 
que establecían con las personas de su entorno más cercano, las cuales proporcionaban soluciones a los problemas y quehaceres cotidianos ${ }^{(2)}$. Estas personas que actualmente se encuentran en la tercera edad han vivido situaciones en las que era frecuente acudir a la casa del único vecino que contaba con teléfono y aparato de radio en su domicilio para recibir una llamada urgente, poder dar aviso para ser atendido por los sanitarios de la zona o incluso escuchar la radio ${ }^{(1)}$.

Actualmente nos encontramos inmersos en la era digital, en la que tanto el uso de internet y teléfono móvil es parte de la vida, e incluso fundamental para mantener y potenciar las relaciones sociales sin olvidar los beneficios a nivel cognitivo y psicológico que produce su buen uso en el colectivo en las personas mayores, evitando el aislamiento social ${ }^{(3)}$.

Centrándonos en España, se puede comentar que el aumento de este grupo poblacional se debe principalmente a la disminución de la mortalidad y al aumento de la esperanza de vida, sin olvidar la disminución de la natalidad y los fenómenos migratorios. Por tanto, se puede hablar de un crecimiento espectacular en el número de personas mayores de 65 años, y por ende, un aumento de la necesidad de los recursos sanitarios para enfermedades crónicas, cuestión que lleva implícito personal sanitario especializado en geriatría $^{(4)}$.

Incluso, estos profesionales deben de incorporar las nuevas tecnologías como recurso sanitario para atender a este creciente sector de la sociedad, utilizándose éstas para programas de promoción de la salud y aumento en su calidad de vida. Estos cambios acontecidos repercuten a nivel social, sanitario y cultural, así como en la calidad de vida de las personas mayores de 65 años $^{(4)}$.

El hecho de que este grupo de edad pueda participar en actividades sociales y culturales supone que se les permita una continuidad tanto mental como social una vez llegado el final de su etapa laboral. Como consecuencia, estas personas se mantienen activas tras su jubilación y mejora su salud, sobre todo a nivel psicológico. Se puede afirmar que actualmente los medios digitales, los cuales son más utilizados por las poblaciones jóvenes, van en aumento dentro de su uso en la población más envejecida, cuestión que conlleva una mejora en la autoestima e incluso la sensación de mayor autorrealización ${ }^{(5)}$. Por tanto, esta herramienta proporciona una cobertura sociosanitaria, una continuidad del rol social tras su jubilación y como consecuencia, una mayor sensación de felicidad ${ }^{(4-5)}$.

Dada la evidencia científica de la mejora de salud en este grupo poblacional por el uso de las nuevas tecnologías, se hace imprescindible el estudio de éstas en los profesionales sanitarios. Por este motivo, en el Grado de Enfermería, y aplicando las competencias transversales del Plan Bolonia, se aprende el manejo de las herramientas TIC. Tras esto, los futuros enfermeros/as podrán realizar una labor educativa y promoción de la salud dentro de la Atención Primaria sobre el uso de las nuevas tecnologías a los ancianos, haciendo especial hincapié en aquellas aplicaciones relacionadas con la salud. Éstas pueden ser un apoyo donde este grupo poblacional registre información vital para el seguimiento de sus enfermedades crónicas, tales como niveles de glucemia, tensión arterial, actividad física realizada. Esto convierte a las nuevas tecnologías no solo en un apoyo social para los ancianos, sino en recurso que puede mejorar su salud ${ }^{(6)}$.

La educación a las personas mayores de 65 años en cuestión de nuevas tecnologías (TIC) podrían mejorar su calidad de vida, en áreas como las relaciones sociales, las comunicaciones, la mejora de su memoria y capacidad de aprendizaje. Como consecuencia, no solo aumenta la esperanza de vida, sino también la calidad de ésta. Las instituciones, tanto privadas como públicas, deben potenciar el aprendizaje del uso de las nuevas tecnologías a este grupo poblacional. En relación al rol social de las personas de la tercera edad, podemos definir dos grupos de apoyo: el primario (familia, amigos, vecinos, entre otros) y el secundario (relacionado con las nuevas tecnologías). Por tanto, se puede afirmar que el uso de las redes sociales unido al envejecimiento, puede disminuir el sentimiento de soledad, puesto que es una red de apoyo para este grupo etario ${ }^{(1)}$. 
Es de vital importancia potenciar el envejecimiento activo, el cual la Organización Mundial de la Salud (OMS), define como el "proceso de aprovechar al máximo las oportunidades para tener el bienestar físico, psíquico y social durante toda la vida" ${ }^{(7)}$. En consecuencia, se debe de promover políticas sociosanitarias que promuevan un envejecimiento activo, por parte de los ministerios con competencia en salud y de organizaciones internacionales, e incluso por la sociedad civil. La finalidad es la mejora de la salud, la participación y la utilización de nuevas metodologías activas incluidas dentro de las TIC en las personas mayores ${ }^{(2)}$.

A modo de síntesis, se puede afirmar que el uso de las nuevas tecnologías se da desde la educación primaria, incluso a nivel universitario. Por tanto, la población más joven utilizan éstas como un divertimento y como una herramienta de trabajo ${ }^{(8)}$. Por el contrario, la población de la tercera edad lo suelen utilizar en la mayoría de las ocasiones para mantener relaciones familiares y también con sus iguales.

Los teléfonos móviles poseen características y funciones, las cuales han generado en estos mayores el acceso a libros electrónicos, el responder correos, enviar mensajes cortos e incluso jugar on-line. Algunos de los motivos explorados para el uso de la telefonía móvil ha tenido que ver con el entretenimiento y alivio del estrés, e incluso abrir nuevas capacidades de actuación, ya que promueven la creatividad y la expresión, de manera que se ha convertido en una práctica cotidiana a efectos de interacción social y relajación ${ }^{(7)}$.

A su vez, se ha encontrado que, ante la aparición de acontecimientos vitales estresantes, el uso de las tecnologías ayuda a gestionar el estado de ánimo, incluso ganar reconocimiento ante iguales y potenciar las relaciones sociales ${ }^{(3)}$. Así, las personas mayores consumidoras tanto de internet como de telefonía móvil, se ha valorado una menor incidencia de problemas a nivel mental, e índices mayores de participación e integración social con sus iguales.

\section{Referencias:}

1. Martínez Ortega MP, Polo Luque ML, Carrasco Fernández B. Visión histórica del concepto de vejez desde la Edad Media. 2002;6(11):40-6.

2. Lazo MM. Uso de las nuevas tecnologías por las personas mayores en una localidad rural. Vivat Academia. 2015;18(130):21-36.

3. Nef T, Ganea RL, Müri RM, Mosimann PU. Social networking sites and older users - a systematic review. Int Psychogeriat. 2013;25(7):1041-53.

4. Gómez-Cantarino S, Duque MC, Sukkariech Noria S. La formación multiprofesional en la formação sanitaria especializada: otras unidades multiprofesionales en la formación sanitaria especializada desde el punto de vista de la enfermería. In: Millán J, Palés JL, Morán-Barrios J. Principios de Educación Médica. Madrid: Panamericana; 2015. p. 438-44

5. Espina-Jerez B, Gómez-Cantarino S, Sim-Sim M. Smartphone: It's Use for Stress Coping in Spanish Teenagers. Am J Appl Psychol; 2019;8(1):8-13.

6. Queirós PJP, Almeida Filho AJ, Oliveira AC, Gómez-Cantarino S, Almeida FAB, Pascoal JRM. Nursing in the logbooks of sessions of the Houses of the Portuguese monarchy, between 1900 and 1910. Rev Enf Ref. 2019;4(22):149-59.

7. Wang JL, Wang HZ, Gaskin J, Wang LH. The role of stress and motivation in problematic smartphone use among college students. Comput Hum Behav. 2015;(53):181-8.

8. Casado Muñoz R, Lezcano Barbero F, Rodríguez Conde MJ. Envejecimiento activo y acceso a las tecnologías: Un estudio empírico evolutivo. Comunicar. 2015;23(45):37-46. 
Recebido: 9 de março de 2020

Aprovado: 9 de março de 2020

Publicado: 16 de abril de 2020

A Revista Baiana de Enfermagem utiliza a Licença Creative Commons - Atribuição-NãoComercial 4.0 Internacional. https://creativecommons.org/licenses/by-nc/4.0/ Este artigo é de acesso aberto distribuído sob os termos da Licença Creative Commons (CC BY-NC). Esta licença permite que outros remixem, adaptem e criem a partir do seu trabalho para fins não comerciais. Embora os novos trabalhos tenham de lhe atribuir o devido crédito e não possam ser usados para fins comerciais, os usuários não têm de licenciar esses trabalhos derivados sob os mesmos termos. 